
3 Research Square
Preprints are preliminary reports that have not undergone peer review.
They should not be considered conclusive, used to inform clinical practice,
or referenced by the media as validated information.

\title{
RNA-sequencing reveals the expression profiles of tsRNAs and their potential carcinogenic role in cholangiocarcinoma
}

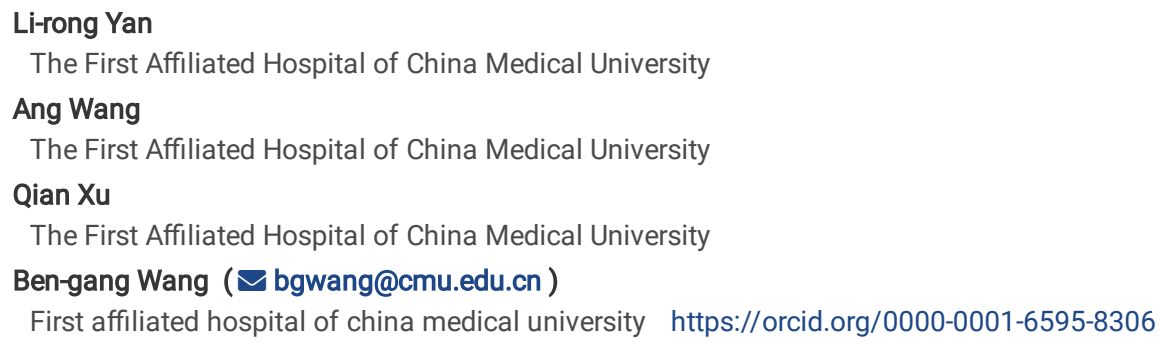

The First Affiliated Hospital of China Medical University

Ang Wang

The First Affiliated Hospital of China Medical University

Qian Xu

The First Affiliated Hospital of China Medical University

Ben-gang Wang ( $\sim$ bgwang@cmu.edu.cn )

First affiliated hospital of china medical university https://orcid.org/0000-0001-6595-8306

\section{Research Article}

Keywords: Cholangiocarcinoma, tsRNAs, tRFs, tiRNAs, RNA-seq

Posted Date: May 3rd, 2021

DOI: https://doi.org/10.21203/rs.3.rs-35126/v2

License: (a) (1) This work is licensed under a Creative Commons Attribution 4.0 International License. Read Full License 


\section{Abstract \\ Background}

Recently, the incidence of cholangiocarcinoma (CCA) has gradually increased. As CCA has a poor prognosis, the ideal survival rate is scarce for patients. The abnormal expressed tsRNAs may regulate the progression of a variety of tumors, and tsRNAs is expected to become a new diagnostic biomarker of cancer. However, the expression of tsRNAs is obscure and should be elucidated in CCA.

\section{Methods}

High-throughput RNA sequencing technology (RNA-seq) was utilized to determine the overall expression profiles of tsRNAs in 3 pairs CCA and adjacent normal tissues and to screen the tsRNAs that were differentially expressed. The target genes of dysregulated tsRNAs were predicted and the biological effects and potential signaling pathways of these target genes were explored by Gene Ontology (GO) and Kyoto Encyclopedia of Genes and Genomes (KEGG) pathway enrichment analyses. Quantitative real-time polymerase chain reaction (qRT-PCR) was used to validate 11 differentially expressed tRFs with 12 pairs CCA and adjacent normal tissues.

\section{Results}

High-throughput RNA-seq totally demonstrated 535 dysregulated tsRNAs, of which 241 tsRNAs were upregulated and 294 tsRNAs were downregulated in CCA compared with adjacent normal tissues ( $\mid$ log2 (fold change) $\mid \geq 1$ and $P$ value $<0.05$ ). GO and KEGG enrichment analyses indicated that the target genes of dysregulated tRFs (tRF-34-JJ6RRNLIK898HR, tRF-38-0668K87SERM492V and tRF-39-0668K87SERM492E2) were mainly enriched in the Notch signaling pathway, Hippo signaling pathway, CAMP signaling pathway and in growth hormone synthesis, secretion and action, etc. qRT-PCR result showed that tRF-34JJ6RRNLIK898HR/tRF-38-0668K87SERM492V/tRF-39-0668K87SERM492E2 was down-regulated $(P=0.021)$ and tRF-20-LE2WMK81 was up-regulated in $\operatorname{CCA}(P=0.033)$.

\section{Conclusion}

Differentially expressed tRFs in CCA are enriched in many pathways associated with neoplasms, which may impact the tumor progression and have potential to be diagnostic biomarkers and therapeutic targets of CCA.

\section{Introduction}

The incidence rate of Cholangiocarcinoma (CCA) is second in malignant tumor of the hepatobiliary system, with hepatocellular carcinoma as the most often (1). Recently, the global occurrence of CCA has gradually increased, and China is one of the countries with the highest incidence (1-3). With low five-year survival rates, the global cumulative mortality rate of CCA patients has increased markedly (4-7). Hepatocellular carcinoma (HCC) and intrahepatic cholangiocarcinoma are mainly treated by surgical resection. However, many patients are diagnosed at an advanced stage, commonly resulting in recurrence after resection(8). There are many studies on the etiology of HCC but few on CCA. Comprehending the pathogenesis of CCA will be based on the exploration of the etiology of CCA. In addition, the study of the early diagnostic biomarkers of CCA is of great significance for improving the early diagnosis rate and survival rate of patients with CCA.

tRNA-derived small RNAs (tsRNAs) is a kind of small noncoding RNA that is produced by specific enzymes that cleave specific sites of tRNA. According to the different sites of enzyme cleavage, tsRNAs mainly include two parts of tRNA-derived fragments (tRFs) and tRNA halves (tiRNA) (9). tRFs can be consisted of i-tRF, tRF-1, tRF-2, tRF-3, and tRF-5. 5'-tiRNA and 3'-tiRNA belong to tiRNA (10). Recently, many features of tsRNAs have been explored, mainly in terms of the following two aspects. On the one hand, some tRFs can bind to the Argonaute protein, an important member of the RNA-induced silencing complex, in a manner similar to miRNA or piRNA, to perform biological functions(11). Furthermore, tRFs can directly regulate protein translation at the post transcriptional level: 5'-tiRNA (5'-tiRNA ${ }^{\text {Ala }}$ and 5'-tiRNA ${ }^{\text {Cys }}$ ) containing 5'-terminal oligoguanine motifs can replace elF4F (elF4F is a eukaryotic transcription initiation factor) on the m7GTP of mRNA, thus inhibiting translation initiation and generating multiple mRNA protein complexes (mRNPs)(12) to directly regulate protein translation. In addition, it has been reported that tsRNAs are critical to the initiation and development of tumors and have influenced on the carcinogenesis of tumors. Some researchers have demonstrated that there are four tsRNAs, including AS-tDR-000064, AS-tDR-000069, AS-tDR-000102 and AS-tDR-001391, can be upregulated in pancreatic carcinoma by using RNA-seq(13). Further GO and KEGG enrichment analysis showed that these four tsRNAs differentially expressed in pancreatic cancer are enriched in diverse pathways associated with cancer, such as 'the PI3K/Akt pathway', 'the Ras pathway', and 'axon guidance', and have the potential to become a new biomarker of pancreatic carcinoma(13). Moreover, one study indicated that tRF-LeuCAG was remarkably upregulated in the serum, cells, and tissues of non-small-cell lung cancer, and tRF-Leu-CAG could contribute to cells proliferation and maintain cell cycle of lung cancer cells, which suggested that tRF-Leu-CAG made a difference in the initiation and development of lung cancer(14).

At present, it has been disclosed that tsRNAs can participate in the development of all kinds of tumors(10), but it is still unclear whether tsRNAs has an impact on the origin of CCA. Exploration of the changes in tsRNAs in CCA compared with normal control tissues could be of great significance in determining early diagnostic markers of CCA. 
In this study, we collected CCA and adjacent normal tissues from three patients and detected the overall expression profiles of tsRNAs in the two groups by high-throughput RNA sequencing (RNA-seq) and further analysis and screening of differentially expressed tsRNAs. Subsequently, the target genes of differentially expressed tRFs were predicted. Gene Ontology (GO) and Kyoto Encyclopedia of Genes and Genomes (KEGG) analyses were taken advantage of predicting the important biological roles and potential signal pathways of differentially expressed tsRNAs in CCA. Quantitative real-time polymerase chain reaction (qRT-PCR) was used to validate the differentially expressed tRFs with CCA and adjacent normal tissues. In general, the main intention of our research was to detect the occurrence and etiology of CCA, to identify early diagnostic biomarkers of CCA and to provide new molecular targets for the remedy of CCA.

\section{Materials And Methods}

\subsection{Patients and samples}

Our research was implemented according to the Declaration of Helsinki and supported by the research ethics committee of the First Affiliated Hospital of 'China medical university'. The written informed consents of our study were signed by all patients. We gathered CCA and adjacent normal tissues of 15 patients at the First Affiliated Hospital of 'China medical university' between September and December 2020. The patient information is listed in Supplementary Table 1. After collection, the samples were frozen in RNAlater (Life Technologies, Carlsbad, California, USA) storage solution and stored at -80 ${ }^{\circ} \mathrm{C}$ for RNA extraction. All specimens were confirmed to be CCA by histopathology. All patients were not treated with radiotherapy, chemotherapy or targeted therapy before operation.

\subsection{Preparation ofRNA Library and RNAsequencing}

Three pairs of tissues from CCA patients were used to acquire the extract of total RNA. High throughput sequencing technology was supplied by CloudSeq Biotech (Shanghai, China). The tRF\&tiRNA sequencing library was generated by total RNA of each tissue. The steps of library preparation mainly included 3'adaptor ligation; 5'-adaptor ligation; synthesis of cDNA; PCR amplification; recovery of $\sim 150 \mathrm{bp}$ PCR amplification fragment. The libraries were denatured as single-stranded DNA molecules. The Illumina flow cells were used to capture single-stranded DNA molecules and subsequently these molecules were amplified into clusters in situ. Finally, the sequence of 50 cycles was implemented by Illumina HiSeq sequencer following the supplier's directions.

\subsection{Data analysis}

The steps of generation raw data mainly included sequencing, analysis of image, base calling and quality filtering, which were carried out by Illumina sequencer. Firstly, quality control was executed by Q30. Cutadapt software (v1.9.3) was used to trim the adaptor sequences and select the adaptor-trimmedreads ( $\geq 16 \mathrm{nt}$ ). Then, the raw counts of each tRF\&tiRNA (MINTbase v2.0) was calculated for all samples, considered as the amount of original expression of that tRF\&tiRNA. EdgeR software (v3.16.5) was utilized to standardize the raw counts and differentially expressed tRF\&tiRNAs between two groups were calculated by edgeR and filtered by Fold Change and P-value. Raw data were uploaded to Gene Expression Omnibus (GEO), GSE147017.

\subsection{Target gene prediction of differentially expressed tRFs}

Similar to miRNAs, tsRNAs also can bind to Argonaute proteins $(15,16)$. It's reported that tRFs, similar to miRNAs, can target mRNA(16-18). Furthermore, some studies suggested that the seed sequences of tRFs have spectra analogous to those of miRNAs $(16,19,20)$. In view of these findings, the target genes of the screened tRFs were predicted by miRanda (http://www.microrna.org/microrna/home.do; August 2010 release), which mainly depend on the binding sites existing in the $3^{\prime}$-untranslated region (3'-UTR). The tRFs that targeted genes more than 90 was included in subsequent analysis. Cytoscape 3.7.2 was used to visualize. All analysis were performed by RStudio 3.6.1.

\subsection{Enrichment analysis of tRF's target genes}

Subsequently, the clusterProfiler R package was used to perform GO (biological process, molecular function and cellular component) and KEGG enrichment analysis for the target genes of tRFs. The results were visualized by Cytoscape 3.7.2. The cutoff criteria is p.adjustment values $<0.05$ and $q$ values $<0.05$.

\section{6 qRT-PCR validation}

Differentially expressed tRFs were selected to perform qRT-PCR validation. Total RNA was extracted from 12 pairs CCA and adjacent normal samples by TRIZOL (TIANGEN BIOTECH). Total RNA was pretreated by rtStar ${ }^{\mathrm{TM}}$ tRF\&tiRNA Pretreatment Kit (Cat\# AS-FS-005). cDNA was synthesized with the itStar ${ }^{\text {TM }}$ First-Strand cDNA Synthesis Kit (3' and 5' adaptor) (Cat\#AS-FS-003-02, Arraystar, MD 20850, USA). The primers were designed for Predesigned Human tRNA Primer Sets V2.0. U6 was utilized as an internal control. Quantitative Real-time PCR was performed by Arraystar SYBR Green Real-Time qPCR Master Mix (Cat\#AS-MR-006-5). The relative expression level of each tRNA-derived fragments was calculated with $2^{-\triangle C t}$. SPSSv24.0 (IBM, SPSS, and Chicago, IL, USA) and GraphPad Prism V8.0 (GraphPad software, USA) were used to perform statistical analysis. $P<0.05$ was statistically significant.

\section{Results}

\subsection{Expression profiles of tsRNAs in CCA}

The results indicated that RNA-seq totally detected 20102 tsRNAs in the two groups, of which 9616 were up-regulated and 10486 were down-regulated. Difference of tsRNAs expression between CCA and adjacent normal tissues were displayed by scatter plot (Figure 1). The dysregulated tsRNAs were selected under the condition of $\mid \log 2$ (fold change) $\mid \geq 1$ and $P$ value $<0.05$. We found out 535 differentially expressed tsRNAs in this study. We discovered that the up- 
regulated and down-regulated of tsRNAs were 241 (Supplementary Table 2) and 294 (Supplementary Table 3) respectively in the CCA tissues. The volcano map showed a significant difference in tsRNAs in the CCA (Figure 2). The results of hierarchical clustering demonstrated different expression of tsRNAs between CCA and adjacent normal tissues (Figure 3). Table 1 and Table 2 displayed the top 30 tsRNAs that were significantly upregulated and downregulated, respectively.

\subsection{Identification target genes of differentially expressed tRFs}

Based on miRanda, the target genes of differentially expressed tRFs were predicted. The tRFs with target genes more than 90 was included in subsequent analysis. Finally, the result demonstrated that only 3 differentially expressed tRFs met the inclusion criteria, including tRF-38-0668K87SERM492V, tRF-390668K87SERM492E2 and tRF-34-JJ6RRNLIK898HR. The differentially expressed information of these tRFs were listed in Table 3. The target genes with tRFs were visualization in Figure 4.

\subsection{Enrichment analyses revealing the correlation of tRFs with CCA}

Subsequently, the target genes of 3 tRFs were performed GO and KEGG analysis by clusterProfiler R package. tRF-38-0668K87SERM492V enrichment analysis showed that it was related to formulate cellular transport complex, regulate different voltage-gated channels activity and participate in Hippo signaling pathway and Notch signaling pathway (Figure 5A). tRF-39-0668K87SERM492E2 was also associated with different voltage-gated channels activity, Hippo signaling pathway and Notch signaling pathway (Figure 5B). tRF-34-JJ6RRNLIK898HR was mainly involved in epithelial cell differentiation and participated in CAMP signaling pathway and growth hormone synthesis, secretion and action (Figure 5C). The relationships between target genes and pathways are visualized in Figure 6.

\section{4 qRT-PCR validation of differentially expressed tRFs}

A total of 11 differentially expressed tRFs were selected to perform qRT-PCR validation by 12 pairs CCA tissues. The result showed that tRF-34JJ6RRNLIK898HR/tRF-38-0668K87SERM492V/tRF-39-0668K87SERM492E2 was down-regulated ( $P=0.021$, Figure 7A) and tRF-20-LE2WMK81 was upregulated in $\mathrm{CCA}(P=0.033$, Figure $7 \mathrm{~B})$, which were consistent with our RNA-seq results. Other validated molecules have no statistical significance, including tRF-19-R118LOJX (Figure 8A), tRF-19-BR29N3E2 (Figure 8B), tRF-21-RK9P4P9L0 (Figure 8C), tRF-16-9NF5W8B (Figure 8D), tRF-16-KWEKK1B (Figure 8E), tRF17-K5KKOV2/tRF-18-P6KP6HD2 (Figure 8F), tRF-31-6978WPRLXN4VE/tRF-22-8B8SOUPR2 (Figure 8G), tRF-18-1SS2P4X/tRF-17-YEKPRSP/tRF-17ML5FX23/tRF-16-V0J809E (Figure 8H).

\section{Discussion}

Noncoding RNAs(ncRNAs) are consisted of microRNAs (miRNAs), long ncRNAs (IncRNAs), circular RNAs (circRNAs), and tsRNAs, which are small RNAs that are not capable of encoding proteins $(9,21,22)$. Recently, with the application of high-throughput sequencing technology and biological information analyses in ncRNAs, researchers have revealed many new research theories, mainly including the theory that ncRNAs such as tsRNAs (including tRFs and tiRNAs) are of great importance in the initiation and development of tumors and can be used as molecular markers for tumor diagnosis or molecular targets for treatment.

Researches have proved that circRNAs and IncRNAs can be considered molecular markers of tumor diagnosis or therapeutic targets(23, 24). Similarly, tsRNAs can also act as molecular markers of disease diagnosis or molecular targets of treatments $(25,26)$. However, there is little information about differentially expressed tRFs in CCA. Therefore, in our study, we explored the differentially expressed tRFs in CCA by RNA-seq and further analyzed the potential function and biological role of tRFs. In addition, we employed qRT-PCR identify the differentially expressed tRFs with CCA tissues.

In this study, we initially detected 241 up-regulated and 294 down-regulated tsRNAs of CCA tissues by RNA-seq ( $\mid$ log 2 (fold change) $\mid \geq 1$ and $P$ value $<0.05$ ). It's reported that the CD5+ diffuse large B-cell lymphoma, chronic lymphocytic leukemia, lung cancer and breast cancer were found to have differentially expressed tRFs and could have an influence on tumor progression(27-29). We found the differentially expressed tRFs in CCA tissues for the first time. Analogous to results of differentially expressed tRFs before, we speculated that differentially expressed tRFs may have an impact on the progression of tumor and have potential to be diagnostic biomarkers of CCA.

Subsequently, the target genes of differentially expressed tRFs were predicted and were carried out GO and KEGG enrichment analysis. The result revealed that three tRFs (tRF-34-JJ6RRNLIK898HR, tRF-38-0668K87SERM492V, tRF-39-0668K87SERM492E2) were downregulated, and their target genes were enriched in cancer-related pathways $(P<0.05$, adjusted $P<0.05)$. There are many similarities in the three differentially expressed tRFs screened. For instance, although the UniqueIDs are different in MINTbase, they share the same sequence (CAGGCGGCCCGGGTTCGACTCCCGGTGTGGGAAC), and similar regions of chromosomes 13 and 15 can be cut to generate these sequence fragments. These are interesting phenomena that we found in this research, but the specific mechanism remains to be further studied in the future. The target genes of dysregulated tRFs in CCA were mainly enriched in the Notch signaling pathway, Hippo signaling pathway, and cAMP signaling pathway and in growth hormone synthesis, secretion and action, etc. It has been reported that when the cAMP signaling pathway was activated, it may suppress the migration of breast cancer cells (30). The Hippo signaling pathway are relevant to the procession of hepatocellular carcinoma(31), breast carcinoma (32), and gastric carcinoma (33), influencing the proliferation, differentiation, and migration of tumor cells. The Notch signaling pathway is significant to the development of diversified cancers and can regulate the growth, survival, apoptosis, invasion and migration of all kinds of tumor cells, such as pancreatic carcinoma and liver cancer cells (34-37). In addition, research has identified the relationship between tRFs and the Notch signaling pathway and shown that tRF/miR-1280 can bind with the 3'-UTR of JAG2 mRNA to decrease the synthesis of JAG2 (a ligand of Notch signaling pathway), thereby inactivating the Notch signaling pathway and reducing the proliferation and metastasis of CRC (38). tRF/miR-1280 can also depress the Notch signaling pathway, thus reducing the expression level of the CD133+ stem cell phenotype in CRC cells, decreasing tumor motility and 
migration ability and hindering the formation of a tumor metastasis-favorable microenvironment(38). Furthermore, the Notch signaling pathway is great of significance in the carcinogenesis of CCA. Some research claimed that the level of cyclin E protein is positive regulated by the Notch signaling pathway in CCA (39). Some scholars have indicated that when the Notch signaling pathway is activated, it can prompt hepatic progenitor cells and differentiate hepatocytes to turn into biliary lineage cells, thus promoting the carcinogenesis of $\mathrm{CCA}(40)$. The Notch signaling pathway may be a main driving factor of human CCA occurrence and a perspective molecular target of therapy(40). Our study found that the target genes of dysregulated tRFs in CCA could enrich in different cancer-related pathway. According to the study above, we speculated that the dysregulated tRFs in CCA may result in the progression of CCA by regulating these cancer-related enriched pathways. However, its effect on the biological behavior of tumor cells and its specific mechanism needs to be further explored.

qRT-PCR was utilized to identify randomly selected 11 differentially expressed tRFs with 12 pairs CCA tissues. The result illustrated that only tRF-34JJ6RRNLIK898HR/tRF-38-0668K87SERM492V/tRF-39-0668K87SERM492E2 and tRF-20-LE2WMK81 were consistent with RNA-seq data and have statistical significance $(P<0.05)$. The result hinted again that these tRFs may paly important role in CCA progression and is more likely to be diagnostic and prognostic biomarker as well as therapeutic targets.

In summary, we used high-throughput RNA-seq to determine differentially expressed tsRNAs in CCA and adjacent normal tissue. Subsequent GO and KEGG analyses of dysregulated tsRNAs in the two groups indicated that the target genes of tsRNAs were enriched in multiple cancer-related pathways. qRT-PCR also validated the differentially expressed tRF-34-JJ6RRNLIK898HR/tRF-38-0668K87SERM492V/tRF-39-0668K87SERM492E2 and tRF-20-LE2WMK81. Our study suggests that tsRNAs may make a difference in the origin of CCA, which may contribute us to discover the etiological mechanism about CCA, and that tsRNAs may become a new molecular marker of diagnosis and a molecular target of therapy in CCA. This study built a solid foundation for the further pathogenic mechanism research of tRFs in CCA.

\section{Declarations}

\section{Ethics approval and consent to participate}

The study was implemented according to the Declaration of Helsinki and supported by the research ethics committee of the First Affiliated Hospital of 'China medical university'. The written informed consents in this study were signed by all patients.

\section{Consent for publication}

Not applicable.

\section{Availability of data and materials}

The data that support the results of this manuscript are available from the corresponding author upon reasonable request.

\section{Competing interests}

All authors disclose no conflicts of interest that might bias their work.

\section{Funding}

This work is supported partly by grants from the Natural Science Foundation of Liaoning Province in China (Ref No. 20170541001).

\section{Authors' contributions}

Ben-gang Wang conceived and designed this study. Li-rong Yan preformed the experiment. Ang Wang was responsible for the data analysis and performed data interpretation. Li-rong Yan wrote the paper. Qian Xu and Ben-gang Wang revised the manuscript.

\section{Acknowledgements}

We thank American Journal Experts (https://www.aje.com/), for editing the English text of a draft of this manuscript.

\section{Abbreviations}

CCA, Cholangiocarcinoma. tsRNAs, tRNA-derived small RNAs. GO, Gene Ontology. HCC, hepatocellular carcinoma. KEGG, Kyoto Encyclopedia of Genes and Genomes. qRT-PCR, Quantitative real-time polymerase chain reaction.

\section{References}

1. Rizvi S, Khan SA, Hallemeier CL, Kelley RK, Gores GJ. Cholangiocarcinoma - evolving concepts and therapeutic strategies. Nat Rev Clin Oncol. 2018;15(2):95-111.

2. Saha SK, Zhu AX, Fuchs CS, Brooks GA. Forty-Year Trends in Cholangiocarcinoma Incidence in the U.S.: Intrahepatic Disease on the Rise. Oncologist. 2016;21(5):594-9. 
3. Esnaola NF, Meyer JE, Karachristos A, Maranki JL, Camp ER, Denlinger CS. Evaluation and management of intrahepatic and extrahepatic cholangiocarcinoma. Cancer. 2016;122(9):1349-69.

4. Zhang C, Zhang B, Meng D, Ge C. Comprehensive analysis of DNA methylation and gene expression profiles in cholangiocarcinoma. Cancer Cell Int. 2019;19:352.

5. Razumilava N, Gores GJ. Cholangiocarcinoma. The Lancet. 2014;383(9935):2168-79.

6. Everhart JE, Ruhl CE. Burden of digestive diseases in the United States Part III: Liver, biliary tract, and pancreas. Gastroenterology. 2009;136(4):1134-44.

7. Tao EW, Cheng WY, Li WL, Yu J, Gao QY. tiRNAs: A novel class of small noncoding RNAs that helps cells respond to stressors and plays roles in cancer progression. J Cell Physiol. 2020;235(2):683-90.

8. Lee EJ, Chung HW, Jo JH, So Y. Radioembolization for the Treatment of Primary and Metastatic Liver Cancers. Nucl Med Mol Imaging. 2019;53(6):36773.

9. Shen Y, Yu X, Zhu L, Li T, Yan Z, Guo J. Transfer RNA-derived fragments and tRNA halves: biogenesis, biological functions and their roles in diseases. J Mol Med (Berl). 2018;96(11):1167-76.

10. Zhu L, Ge J, Li T, Shen Y, Guo J. tRNA-derived fragments and tRNA halves: The new players in cancers. Cancer Lett. 2019;452:31-7.

11. Short AK, Yeshurun S, Powell R, Perreau VM, Fox A, Kim JH, et al. Exercise alters mouse sperm small noncoding RNAs and induces a transgenerational modification of male offspring conditioned fear and anxiety. Translational Psychiatry. 2017;7.

12. Lyons SM, Achorn C, Kedersha NL, Anderson PJ, Ivanov P. YB-1 regulates tiRNA-induced Stress Granule formation but not translational repression. Nucleic Acids Res. 2016;44(14):6949-60.

13. Jin L, Zhu C, Qin X. Expression profile of tRNA-derived fragments in pancreatic cancer. Oncol Lett. 2019;18(3):3104-14.

14. Shao Y, Sun Q, Liu X, Wang P, Wu R, Ma Z. tRF-Leu-CAG promotes cell proliferation and cell cycle in non-small cell lung cancer. Chemical Biology \& Drug Design. 2017;90(5):730-8.

15. Shigematsu M, Kirino Y. tRNA-Derived Short Non-coding RNA as Interacting Partners of Argonaute Proteins. Gene Regul Syst Bio. 2015;9:27-33.

16. Wang C, Zhao M, Wang J, Zhang D, Wang S, Zhao J. Expression analysis of transfer RNAderived fragments in the blood of patients with moyamoya disease: A preliminary study. Mol Med Rep. 2019;19(5):3564-74.

17. Karaiskos S, Grigoriev A. Dynamics of tRNA fragments and their targets in aging mammalian brain. F1000Research. 2016;5.

18. Haussecker D, Huang Y, Lau A, Parameswaran P, Fire AZ, Kay MA. Human tRNA-derived small RNAs in the global regulation of RNA silencing. RNA. 2010;16(4):673-95.

19. Miyoshi K, Miyoshi T, Siomi H. Many ways to generate microRNA-like small RNAs: non-canonical pathways for microRNA production. Molecular Genetics and Genomics. 2010;284(2):95-103.

20. Maute RL, Schneider C, Sumazin P, Holmes A, Califano A, Basso K, et al. tRNA-derived microRNA modulates proliferation and the DNA damage response and is down-regulated in B cell lymphoma. Proc Natl Acad Sci U S A. 2013;110(4):1404-9.

21. Ledda B, Ottaggio L, Izzotti A, Sukkar SG, Miele M. Small RNAs in eucaryotes: new clues for amplifying microRNA benefits. Cell \& Bioscience. 2020;10(1).

22. Yousefi F, Soltani BM. Circular RNAs as potential theranostics in the cardiac fibrosis. Heart failure reviews. 2020.

23. Li XN, Wang ZJ, Ye CX, Zhao BC, Li ZL, Yang Y. RNA sequencing reveals the expression profiles of circRNA and indicates that circDDX17 acts as a tumor suppressor in colorectal cancer. J Exp Clin Cancer Res. 2018;37(1):325.

24. Han Y, Wang X, Mao E, Shen B, Huang L. Analysis of Differentially Expressed IncRNAs and mRNAs for the Identification of Hypoxia-Regulated Angiogenic Genes in Colorectal Cancer by RNA-Seq. Med Sci Monit. 2019;25:2009-15.

25. Su Z, Kuscu C, Malik A, Shibata E, Dutta A. Angiogenin generates specific stress-induced tRNA halves and is not involved in tRF-3-mediated gene silencing. The Journal of biological chemistry. 2019;294(45):16930-41.

26. Dhahbi JM. 5' tRNA Halves: The Next Generation of Immune Signaling Molecules. Frontiers in Immunology. 2015;6.

27. Qu Q, Li Y, Fang X, Zhang L, Xue C, Ge X, et al. Differentially expressed tRFs in CD5 positive relapsed \& refractory diffuse large B cell lymphoma and the bioinformatic analysis for their potential clinical use. Biology Direct. 2019;14(1).

28. Pekarsky Y, Balatti V, Palamarchuk A, Rizzotto L, Veneziano D, Nigita G, et al. Dysregulation of a family of short noncoding RNAs, tsRNAs, in human cancer. Proc Natl Acad Sci U S A. 2016;113(18):5071-6.

29. Farina NH, Scalia S, Adams CE, Hong D, Fritz AJ, Messier TL, et al. Identification of tRNA-derived small RNA (tsRNA) responsive to the tumor suppressor, RUNX1, in breast cancer. J Cell Physiol. 2020;235(6):5318-27.

30. Dong H, Claffey KP, Brocke S, Epstein PM. Inhibition of breast cancer cell migration by activation of cAMP signaling. Breast Cancer Res Treat. 2015;152(1):17-28

31. Liu AM, Xu MZ, Chen J, Poon RT, Luk JM. Targeting YAP and Hippo signaling pathway in liver cancer. Expert opinion on therapeutic targets. 2010;14(8):855-68.

32. Zhou X, Wang S, Wang Z, Feng X, Liu P, Lv X-B, et al. Estrogen regulates Hippo signaling via GPER in breast cancer. Journal of Clinical Investigation. 2015;125(5):2123-35.

33. Qiao Y, Li T, Zheng S, Wang H. The Hippo pathway as a drug target in gastric cancer. Cancer Lett. 2018;420:14-25.

34. Ntziachristos P, Lim Jing S, Sage J, Aifantis I. From Fly Wings to Targeted Cancer Therapies: A Centennial for Notch Signaling. Cancer Cell. 2014;25(3):318-34

Page 6/15 
35. Cui L, Dong Y, Wang X, Zhao X, Kong C, Liu Y, et al. Downregulation of long noncoding RNA SNHG1 inhibits cell proliferation, metastasis, and invasion by suppressing the Notch-1 signaling pathway in pancreatic cancer. J Cell Biochem. 2019;120(4):6106-12.

36. Dai X, Guo X, Liu J, Cheng A, Peng X, Zha L, et al. Circular RNA circGRAMD1B inhibits gastric cancer progression by sponging miR-130a-3p and regulating PTEN and p21 expression. Aging. 2019;11(21):9689-708.

37. Li J-H, Zhu X-X, Li F-X, Huang C-S, Huang X-T, Wang J-Q, et al. MFAP5 facilitates the aggressiveness of intrahepatic Cholangiocarcinoma by activating the Notch1 signaling pathway. Journal of Experimental \& Clinical Cancer Research. 2019;38(1).

38. Huang B, Yang H, Cheng X, Wang D, Fu S, Shen W, et al. tRF/miR-1280 Suppresses Stem Cell-like Cells and Metastasis in Colorectal Cancer. Cancer Res. 2017;77(12):3194-206.

39. Zender S, Nickeleit I, Wuestefeld T, Sorensen I, Dauch D, Bozko P, et al. A critical role for notch signaling in the formation of cholangiocellular carcinomas. Cancer Cell. 2013;23(6):784-95.

40. Cigliano A, Wang J, Chen X, Calvisi DF. Role of the Notch signaling in cholangiocarcinoma. Expert Opinion on Therapeutic Targets. 2017;21(5):471-83.

\section{Tables}

Table 1 Top 30 up-regulated tRFs in CCA vs. adjacent normal tissues

\begin{tabular}{|c|c|c|c|c|c|c|c|c|}
\hline $\begin{array}{l}\text { que ID } \\
\text { ived) }\end{array}$ & Fragment sequence & $\log \mathrm{FC}$ & Pvalue & FDR & Regulation & \#Туре & $\begin{array}{l}\text { MINTbase Alternative IDs } \\
\text { (GRCh37 assembly-derived) }\end{array}$ & Chromosome \\
\hline VE5D & TTATACCCTTCCCGTACTACC & 5.180160643 & 0.000127872 & 0.991038238 & up & 3'-tRF & trnaMT_MetCAT_MT_+_4402_4469@50.70.21 & MT \\
\hline $3 \mathrm{~B}$ & TGGTTAGCACTCTGGA & 4.765299139 & 0.000127872 & 0.991038238 & up & i-tRF & trna42_GlnCTG_6_+_27263212_27263283@16.31.16 & 6 \\
\hline $3 \mathrm{~B}$ & TGGTTAGCACTCTGGA & 4.765299139 & 0.000127872 & 0.991038238 & up & i-tRF & trna146_GlnCTG_6_-_27515531_27515602@16.31.16 & 6 \\
\hline 3B & TGGTTAGCACTCTGGA & 4.765299139 & 0.000127872 & 0.991038238 & up & i-tRF & trna7_GlnCTG_15_-_66161400_66161471@16.31.16 & 15 \\
\hline $3 \mathrm{~B}$ & TGGTTAGCACTCTGGA & 4.765299139 & 0.000127872 & 0.991038238 & up & i-tRF & trna3_GlnCTG_17_+_8023070_8023141@16.31.16 & 17 \\
\hline $3 \mathrm{~B}$ & TGGTTAGCACTCTGGA & 4.765299139 & 0.000127872 & 0.991038238 & up & i-tRF & trna1_GlnCTG_6_+_18836402_18836473@16.31.16 & 6 \\
\hline 3B & TGGTTAGCACTCTGGA & 4.765299139 & 0.000127872 & 0.991038238 & up & i-tRF & trna49_GlnCTG_6_+_27487308_27487379@16.31.16 & 6 \\
\hline $3 \mathrm{~B}$ & TGGTTAGCACTCTGGA & 4.765299139 & 0.000127872 & 0.991038238 & up & i-tRF & trna99_GlnCTG_6_-_28909378_28909449@16.31.16 & 6 \\
\hline 3B & TGGTTAGCACTCTGGA & 4.765299139 & 0.000127872 & 0.991038238 & up & i-tRF & trna130_GlnTTG_6_-_27763640_27763711@16.31.16 & 6 \\
\hline 3B & TGGTTAGCACTCTGGA & 4.765299139 & 0.000127872 & 0.991038238 & up & i-tRF & trna173_GlnTTG_6_-_26311975_26312046@16.31.16 & 6 \\
\hline $3 \mathrm{~B}$ & TGGTTAGCACTCTGGA & 4.765299139 & 0.002412437 & 0.991038238 & up & i-tRF & trna174_GlnTTG_6_-_26311424_26311495@16.31.16 & 6 \\
\hline 3B & TGGTTAGCACTCTGGA & 4.765299139 & 0.002412437 & 0.991038238 & up & i-tRF & trna64_GlnTTG_6_+_28557156_28557227@16.31.16 & 6 \\
\hline $3 B$ & TGGTTAGCACTCTGGA & 4.765299139 & 0.006323658 & 0.991038238 & up & i-tRF & trna16_GlnTTG_17_+_47269890_47269961@16.31.16 & 17 \\
\hline KWE52 & GTTGGTTATACCCTTCCCGTACTACCA & 5.690385949 & 0.006323658 & 0.991038238 & up & 3'-tRF & trnaMT_MetCAT_MT_+_4402_4469@45.71.27 & MT \\
\hline KP & GGGTATGATTCTCGGTTTG & 5.160191852 & 0.006323658 & 0.991038238 & up & i-tRF & trna12_ProTGG_11_-_75946869_75946940@16.34.19 & 11 \\
\hline $32 \mathrm{I} 7 \mathrm{~L} 7 \mathrm{DV}$ & ATGGGTGGTTCAGTGGTAGAATTCTCGCCTGCC & 4.952823031 & 0.006323658 & 0.991038238 & up & i-tRF & trna35_GlyGCC_1_+_161413094_161413164@3.35.33 & 1 \\
\hline $32 \mathrm{I} 7 \mathrm{~L} 7 \mathrm{DV}$ & ATGGGTGGTTCAGTGGTAGAATTCTCGCCTGCC & 4.952823031 & 0.006323658 & 0.991038238 & up & i-tRF & trna37_GlyGCC_1_+_161420467_161420537@3.35.33 & 1 \\
\hline \$2I7L7DV & ATGGGTGGTTCAGTGGTAGAATTCTCGCCTGCC & 4.952823031 & 0.006323658 & 0.991038238 & up & i-tRF & trna39_GlyGCC_1_+_161427898_161427968@3.35.33 & 1 \\
\hline 32I7L7DV & ATGGGTGGTTCAGTGGTAGAATTCTCGCCTGCC & 4.952823031 & 0.006323658 & 0.991038238 & up & i-tRF & trna41_GlyGCC_1_+_161435258_161435328@3.35.33 & 1 \\
\hline $3217 \mathrm{~L} 7 \mathrm{DV}$ & ATGGGTGGTTCAGTGGTAGAATTCTCGCCTGCC & 4.952823031 & 0.006323658 & 0.991038238 & up & i-tRF & trna2_GlyGCC_21_-_18827107_18827177@3.35.33 & 21 \\
\hline IV & AGCGGAAGCGTGCTGGGCC & 6.121699305 & 0.006323658 & 0.991038238 & up & i-tRF & trna61_MetCAT_6_+_27745664_27745735@14.32.19 & 6 \\
\hline IV & AGCGGAAGCGTGCTGGGCC & 6.121699305 & 0.006984158 & 0.991038238 & up & i-tRF & trna32_MetCAT_1_+_153643726_153643797@14.32.19 & 1 \\
\hline IV & AGCGGAAGCGTGCTGGGCC & 6.121699305 & 0.006984158 & 0.991038238 & up & i-tRF & trna20_MetCAT_17_-_80452597_80452668@14.32.19 & 17 \\
\hline TV & AGCGGAAGCGTGCTGGGCC & 6.121699305 & 0.006984158 & 0.991038238 & up & i-tRF & trna129_MetCAT_6_-_27870271_27870342@14.32.19 & 6 \\
\hline TV & AGCGGAAGCGTGCTGGGCC & 6.121699305 & 0.006984158 & 0.991038238 & up & i-tRF & trna142_MetCAT_6_-_27560600_27560671@14.32.19 & 6 \\
\hline IV & AGCGGAAGCGTGCTGGGCC & 6.121699305 & 0.006984158 & 0.991038238 & up & i-tRF & trna150_MetCAT_6_-_27300764_27300835@14.32.19 & 6 \\
\hline TV & AGCGGAAGCGTGCTGGGCC & 6.121699305 & 0.006984158 & 0.991038238 & up & i-tRF & trna169_MetCAT_6_-_26330529_26330600@14.32.19 & 6 \\
\hline TV & AGCGGAAGCGTGCTGGGCC & 6.121699305 & 0.006984158 & 0.991038238 & up & i-tRF & trna171_MetCAT_6_-_26313352_26313423@14.32.19 & 6 \\
\hline TV & AGCGGAAGCGTGCTGGGCC & 6.121699305 & 0.007054099 & 0.991038238 & up & i-tRF & trna2_MetCAT_6_+_26286754_26286825@14.32.19 & 6 \\
\hline$\underline{\text { TV }}$ & AGCGGAAGCGTGCTGGGCC & 6.121699305 & 0.007054099 & 0.991038238 & up & i-tRF & trna151_ThrCGT_6_-_27271568_27271639@14.32.19 & 6 \\
\hline
\end{tabular}

Table 2 Top 30 down-regulated tRFs in CCA vs. adjacent normal tissues 


\begin{tabular}{|c|c|c|c|c|c|c|c|c|}
\hline $\begin{array}{l}\text { MINTbase Unique } \\
\text { ID (sequence } \\
\text { derived) }\end{array}$ & Fragment sequence & $\log F C$ & PValue & FDR & Regulation & Type & $\begin{array}{l}\text { MINTbase Alternative IDs } \\
\text { (GRCh37 assembly-derived) }\end{array}$ & Chromosome \\
\hline tRF-20-739P8WQ0 & GTGGTTGTAGTCCGTGCGAG & -6.165104314 & $5.10975 \mathrm{E}-05$ & 0.991038238 & down & i-tRF & $\begin{array}{l}\text { trnaMT_GluTTC_MT_-_14674_14742@46.65.20 } \\
\text { trnalookalike8_GluTTC_5_- }\end{array}$ & MT \\
\hline tRF-20-739P8WQ0 & GTGGTTGTAGTCCGTGCGAG & -6.165104314 & $5.10975 \mathrm{E}-05$ & 0.991038238 & down & i-tRF & _93905172_93905240@46.65.20 & 5 \\
\hline tRF-34- & & & & & & 3'- & & \\
\hline JJ6RRNLIK898HR & CAGGCGGCCCGGGTTCGACTCCCGGTGTGGGAAC & -4.753211723 & $5.10975 \mathrm{E}-05$ & 0.991038238 & down & tRF & trna3_GluTTC_13__-45492062_45492133@40.73.34 & 13 \\
\hline $\begin{array}{l}\text { tRF-34- } \\
\text { JJ6RRNLIK898HR }\end{array}$ & & & & & & $\begin{array}{l}3{ }^{\prime}- \\
\text { tRF }\end{array}$ & & 15 \\
\hline $\begin{array}{l}\text { JJ6RRNLIK898HR } \\
\text { tRF-17-VL8RPY5 }\end{array}$ & $\begin{array}{l}\text { CAGGCGGCCCGGGTTCGACTCCCGGTGTGGGAAC } \\
\text { TAGTATCCCCGCCTGTC }\end{array}$ & $\begin{array}{l}-4.753211723 \\
-6.201249994\end{array}$ & $\begin{array}{r}5.10975 \mathrm{E}-05 \\
0.000468303\end{array}$ & $\begin{array}{l}0.991038238 \\
0.991038238\end{array}$ & $\begin{array}{l}\text { down } \\
\text { down }\end{array}$ & $\begin{array}{l}\text { tRF } \\
\text { i-tRF }\end{array}$ & $\begin{array}{l}\text { trna11_GluTTC_15_-_26327381_26327452@40.73.34 } \\
\text { trna5_AspGTC_12_+_98897281_98897352@20.36.17 }\end{array}$ & 12 \\
\hline $\begin{array}{l}\text { tRF-23- } \\
\text { YP9LON4VDP }\end{array}$ & [GGTAGAATTC? & -7.6 & 8303 & 38238 & down & i-tRF & trna & 16 \\
\hline $\begin{array}{l}\text { tRF-23- } \\
\text { YP9LON4VDP }\end{array}$ & TTCAGTGGTAGAATTCTCGCCTG & -7.673641325 & 0.000959116 & 0.991038238 & down & i-tRF & trna25_GlyGCC_16_-_70812114_70812184@11.33.23 & 16 \\
\hline $\begin{array}{l}\text { tRF-23- } \\
\text { YP9LON4VDP }\end{array}$ & TTCAGTGGTAGAATTCTCGCCTG & -7.673641325 & 0.000959116 & 0.991038238 & down & i-tRF & trna68_GlyGCC_1_-_161493637_161493707@11.33.23 & 1 \\
\hline $\begin{array}{l}\text { tRF-23- } \\
\text { YP9LON4VDP }\end{array}$ & TTCAGTGGTAGAATTCTCGCCTG & -7.673641325 & 0.000959116 & 0.991038238 & down & i-tRF & trna19_GlyGCC_16_+_70823410_70823480@11.33.23 & 16 \\
\hline $\begin{array}{l}\text { tRF-23- } \\
\text { YP9LON4VDP }\end{array}$ & TTCAGTGGTAGAATTCTCGCCTG & -7.673641325 & 0.000959116 & 0.991038238 & down & i-tRF & trna24_GlyGCC_16_-_70812942_70813012@11.33.23 & 16 \\
\hline $\begin{array}{l}\text { tRF-23- } \\
\text { YP9LON4VDP }\end{array}$ & TTCAGTGGTAGAATTCTCGCCTG & -7.673641325 & 0.000959116 & 0.991038238 & down & i-tRF & trna5_GlyGCC_17_+_8029064_8029134@11.33.23 & 17 \\
\hline $\begin{array}{l}\text { tRF-23- } \\
\text { YP9LON4VDP }\end{array}$ & TTCAGTGGTAGAATTCTCGCCTG & -7.673641325 & 0.000959116 & 0.991038238 & down & i-tRF & trna19_GlyGCC_2_-_157257659_157257729@11.33.23 & 2 \\
\hline $\begin{array}{l}\text { tRF-23- } \\
\text { YP9LON4VDP }\end{array}$ & TTCAGTGGTAGAATTCTCGCCTG & -7.673641325 & 0.000959116 & 0.991038238 & down & i-tRF & trna128_GlyGCC_6_-_27870686_27870756@11.33.23 & 6 \\
\hline $\begin{array}{l}\text { tRF-23- } \\
\text { YP9LON4VDP }\end{array}$ & TTCAGTGGTAGAATTCTCGCCTG & -7.673641325 & 0.000959116 & 0.991038238 & down & i-tRF & trna35_GlyGCC_1_+_161413094_161413164@11.33.23 & 1 \\
\hline $\begin{array}{l}\text { tRF-23- } \\
\text { YP9LON4VDP }\end{array}$ & TTCAGTGGTAGAATTCTCGCCTG & -7.673641325 & 0.000959116 & 0.991038238 & down & i-tRF & trna37_GlyGCC_1_+_161420467_161420537@11.33.23 & 1 \\
\hline $\begin{array}{l}\text { tRF-23- } \\
\text { YP9LON4VDP }\end{array}$ & TTCAGTGGTAGAATTCTCGCCTG & -7.673641325 & 0.000959116 & 0.991038238 & down & i-tRF & trna39_GlyGCC_1_+_161427898_161427968@11.33.23 & 1 \\
\hline $\begin{array}{l}\text { tRF-23- } \\
\text { YP9LON4VDP }\end{array}$ & TTCAGTGGTAGAATTCTCGCCTG & -7.673641325 & 0.000959116 & 0.991038238 & down & i-tRF & trna41_GlyGCC_1_+_161435258_161435328@11.33.23 & 1 \\
\hline $\begin{array}{l}\text { tRF-23- } \\
\text { YP9LON4VDP }\end{array}$ & TTCAGTGGTAGAATTCTCGCCTG & -7.673641325 & 0.000959116 & 0.991038238 & down & i-tRF & trna2_GlyGCC_21_-_18827107_18827177@11.33.23 & 21 \\
\hline $\begin{array}{l}\text { tRF-39- } \\
\text { EH623K76IR3DR2I2 }\end{array}$ & ACGCGGGAGACCGGGGTTCAATTCCCCGACGGGGAGCCA & -5.821002219 & 0.000959116 & 0.991038238 & down & $\begin{array}{l}3{ }^{\prime}- \\
\text { tRF }\end{array}$ & trna5_AspGTC_12_+_98897281_98897352@37.75.39 & 12 \\
\hline tRF-17-18YKISM & AGTGGTTAGGATTCGGC & -7.30441932 & 0.001194796 & 0.991038238 & down & i-tRF & trna25_GluCTC_2_-_71273488_71273560@14.30.17 & 2 \\
\hline tRF-17-18YKISM & AGTGGTTAGGATTCGGC & -7.30441932 & 0.001194796 & 0.991038238 & down & i-tRF & trna59_GluCTC_1_+_249168447_249168518@14.30.17 & 1 \\
\hline tRF-17-18YKISM & AGTGGTTAGGATTCGGC & -7.30441932 & 0.001194796 & 0.991038238 & down & i-tRF & $\begin{array}{l}\text { trna116_GluCTC_1_- } \\
\_145399233 \_145399304 @ 14.30 .17\end{array}$ & 1 \\
\hline tRF-17-18YKISM & AGTGGTTAGGATTCGGC & -7.30441932 & 0.001194796 & 0.991038238 & down & i-tRF & trna71_GluCTC_1_-_161439189_161439260@14.30.17 & 1 \\
\hline tRF-17-18YKISM & AGTGGTTAGGATTCGGC & -7.30441932 & 0.001194796 & 0.991038238 & down & i-tRF & trna74_GluCTC_1_-_161431809_161431880@14.30.17 & 1 \\
\hline tRF-17-18YKISM & AGTGGTTAGGATTCGGC & -7.30441932 & 0.001194796 & 0.991038238 & down & i-tRF & trna77_GluCTC_1_-_161424398_161424469@14.30.17 & 1 \\
\hline tRF-17-18YKISM & AGTGGTTAGGATTCGGC & -7.30441932 & 0.001194796 & 0.991038238 & down & i-tRF & trna80_GluCTC_1_-_161417018_161417089@14.30.17 & 1 \\
\hline tRF-17-18YKISM & AGTGGTTAGGATTCGGC & -7.30441932 & 0.001194796 & 0.991038238 & down & i-tRF & trna77_GluCTC_6_+_28949976_28950047@14.30.17 & 6 \\
\hline tRF-17-18YKISM & AGTGGTTAGGATTCGGC & -7.30441932 & 0.001194796 & 0.991038238 & down & i-tRF & trna87_GluCTC_6_-_126101393_126101464@14.30.17 & 6 \\
\hline tRF-19-Q1Q89PJZ & GCGCCGCTGGTGTAGTGGT & -7.095420921 & 0.001589483 & 0.991038238 & down & $\begin{array}{l}5 '- \\
\text { tRF }\end{array}$ & trna34_GlyCCC_16_-_686736_686806@1.19.19 & 16 \\
\hline tRF-19-Q1Q89PJZ & GCGCCGCTGGTGTAGTGGT & -7.095420921 & 0.001589483 & 0.991038238 & down & $\begin{array}{l}5 \\
\text { tRF } \\
\end{array}$ & trna27_GlyCCC_2_-_70476123_70476193@1.19.19 & 2 \\
\hline
\end{tabular}

Table 3 tRFs enriched in GO and KEGG

\begin{tabular}{|c|c|c|c|c|c|c|c|c|}
\hline $\begin{array}{l}\text { lase Unique ID } \\
\text { nce derived) }\end{array}$ & Fragment sequence & $\log \mathrm{FC}$ & Pvalue & FDR & Regulation & Type & $\begin{array}{l}\text { MINTbase Alternative } \\
\text { IDs (GRCh37 assembly- } \\
\text { derived) }\end{array}$ & Chromosome \\
\hline J6RRNLIK898HR & CAGGCGGCCCGGGTTCGACTCCCGGTGTGGGAAC & -4.753211723 & 0.045042521 & 0.991038238 & down & $\begin{array}{l}3^{\prime}- \\
\text { tRF } \\
3^{\prime}-\end{array}$ & $\begin{array}{l}\text { trna3_GluTTC_13_- } \\
\text { 45492062_45492133@40.73.34 } \\
\text { trna11_GluTTC_15_- }\end{array}$ & 13 \\
\hline J6RRNLIK898HR & CAGGCGGCCCGGGTTCGACTCCCGGTGTGGGAAC & -4.753211723 & 0.045042521 & 0.991038238 & down & tRF & _26327381_26327452@40.73.34 & 15 \\
\hline 'SERM492E2 & ACCCAGGCGGCCCGGGTTCGACTCCCGGTGTGGGAACCA & -6.392275579 & 0.020148165 & 0.991038238 & down & $\begin{array}{l}3^{\prime}- \\
\text { tRF }\end{array}$ & $\begin{array}{l}\text { trna3_GluTTC_13_- } \\
\text { _45492062_45492133@37.75.39 }\end{array}$ & 13 \\
\hline 'SERM492E2 & ACCCAGGCGGCCCGGGTTCGACTCCCGGTGTGGGAACCA & -6.392275579 & 0.020148165 & 0.991038238 & down & $\begin{array}{l}3^{\prime}- \\
\text { tRF }\end{array}$ & $\begin{array}{l}\text { trna11_GluTTC_15_- } \\
\text { _26327381_26327452@37.75.39 }\end{array}$ & 15 \\
\hline 1668K87SERM492V & ACCCAGGCGGCCCGGGTTCGACTCCCGGTGTGGGAACC & -6.48157606 & 0.016704858 & 0.991038238 & down & $\begin{array}{l}3^{\prime}- \\
\text { tRF }\end{array}$ & $\begin{array}{l}\text { trna3_GluTTC_13_- } \\
\text { _45492062_45492133@37.74.38 }\end{array}$ & 13 \\
\hline 1668K87SERM492V & ACCCAGGCGGCCCGGGTTCGACTCCCGGTGTGGGAACC & -6.48157606 & 0.016704858 & 0.991038238 & down & $\begin{array}{l}3^{\prime}- \\
\text { tRF }\end{array}$ & $\begin{array}{l}\text { trna11_GluTTC_15_- } \\
\text { 26327381_26327452@37.74.38 }\end{array}$ & 15 \\
\hline
\end{tabular}

\section{Figures}




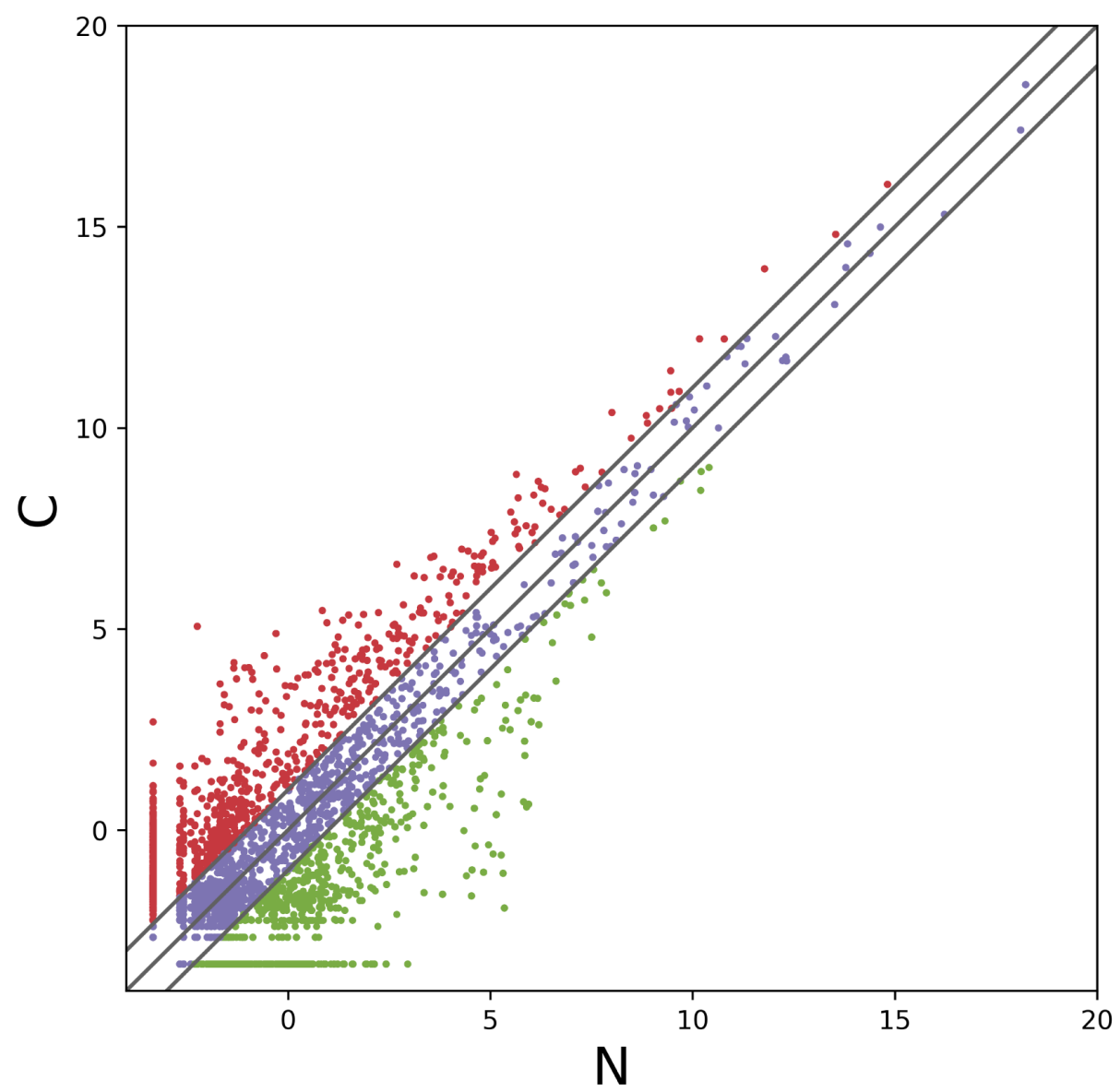

Figure 1

The scatter plot of the differentially expressed tRFs\&tiRNA in CCA and adjacent normal tissues, with red dots indicating up-regulated and green dots indicating down-regulated. The default multiple change threshold is 2.0. CCA, cholangiocarcinoma; tRFs, tRNA-derived fragments; tiRNA, tRNA halves. C, CCA tumor tissues; $\mathrm{N}$, adjacent normal tissues. 


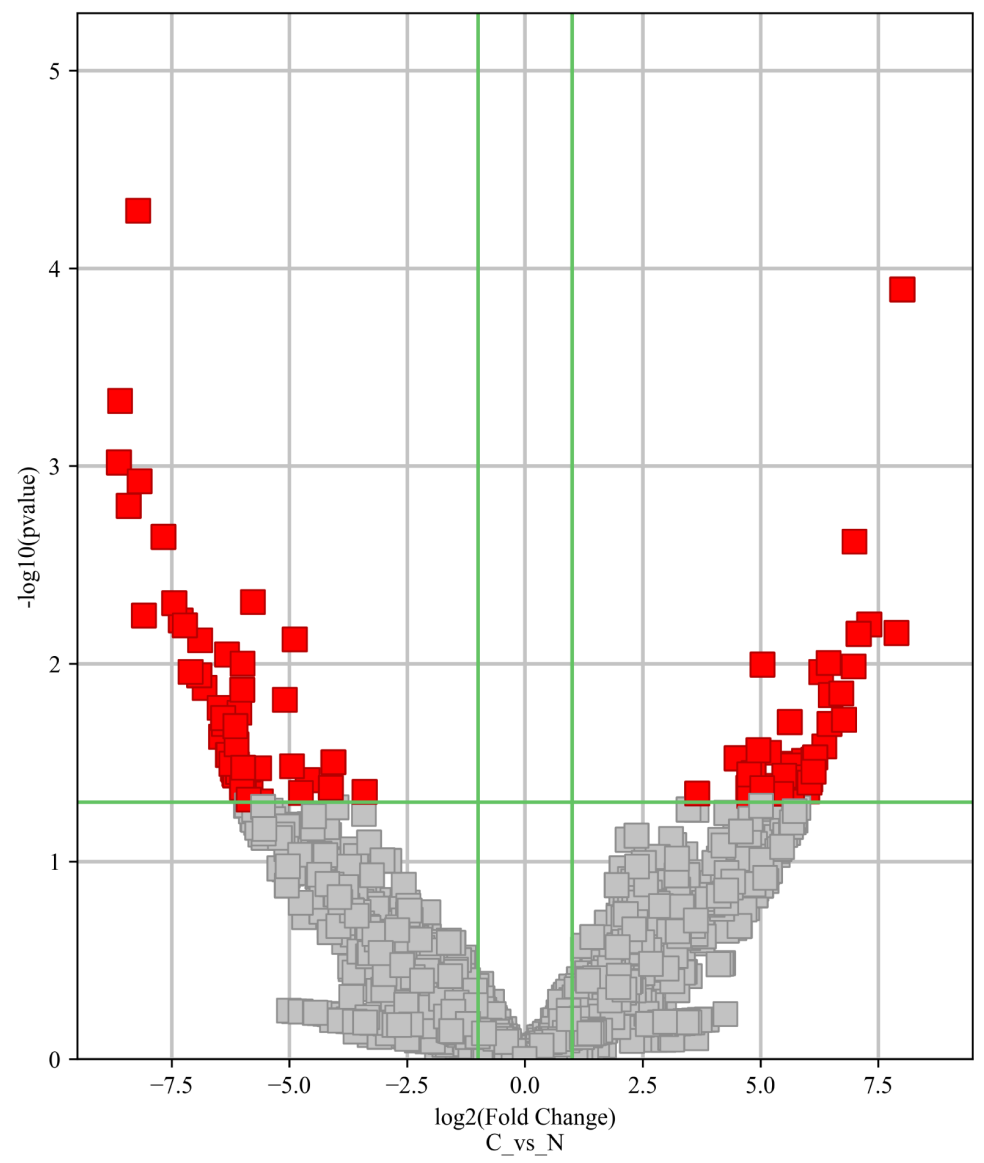

Figure 2

Volcano plot of the differentially expressed tRFs\&tiRNA. Red rectangle represents differential expression of tRFs\&tiRNA in patients with CCA and adjacent normal tissues ( $\mid$ log2 (fold change) $\mid \geq 1 ; P<0.05$ ). CCA, cholangiocarcinoma; tRFs, tRNA-derived fragments; tiRNA, tRNA halves. C, CCA tissues; $N$, adjacent normal tissues. 


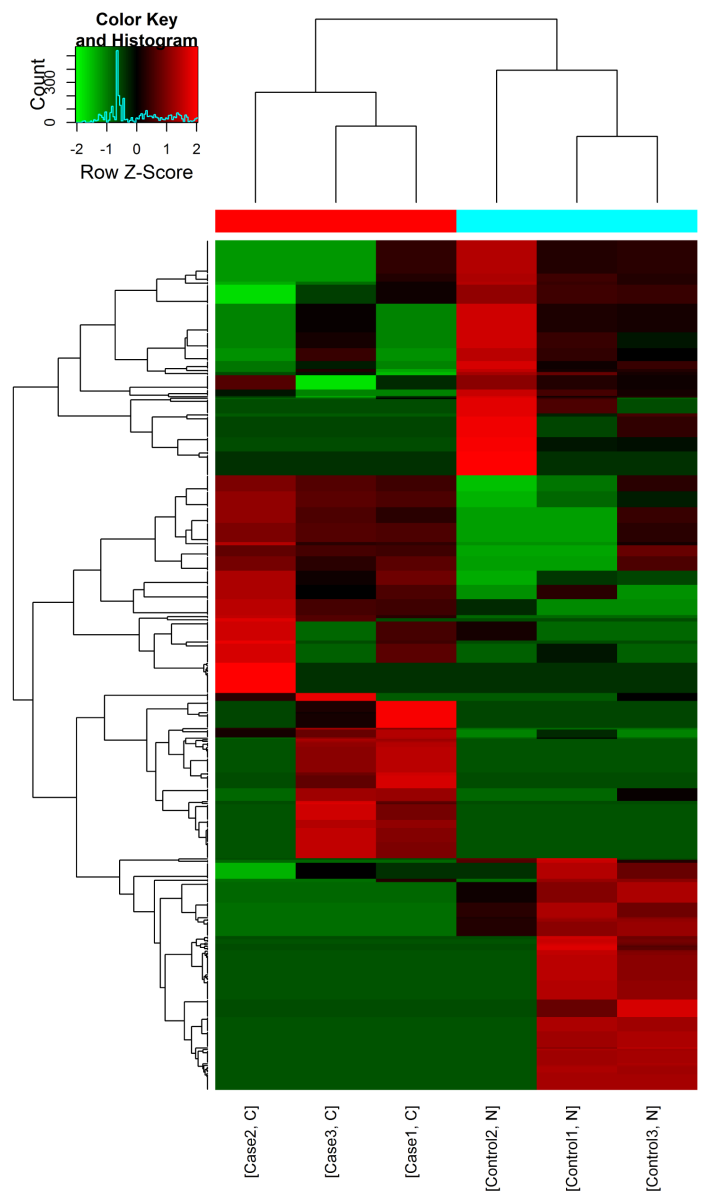

Figure 3

Hierarchical clustering of the differentially expressed tRFs in CCA and adjacent normal tissues. The color scale showed the expression values. The green represented relatively lower expression and red represented relatively higher expression. One tissue sample was represented by each column, and a single tRFs was represented by each row. CCA, cholangiocarcinoma; tRFs, transfer RNA-derived fragments. C, CCA tissues; $\mathrm{N}$, adjacent normal tissues.
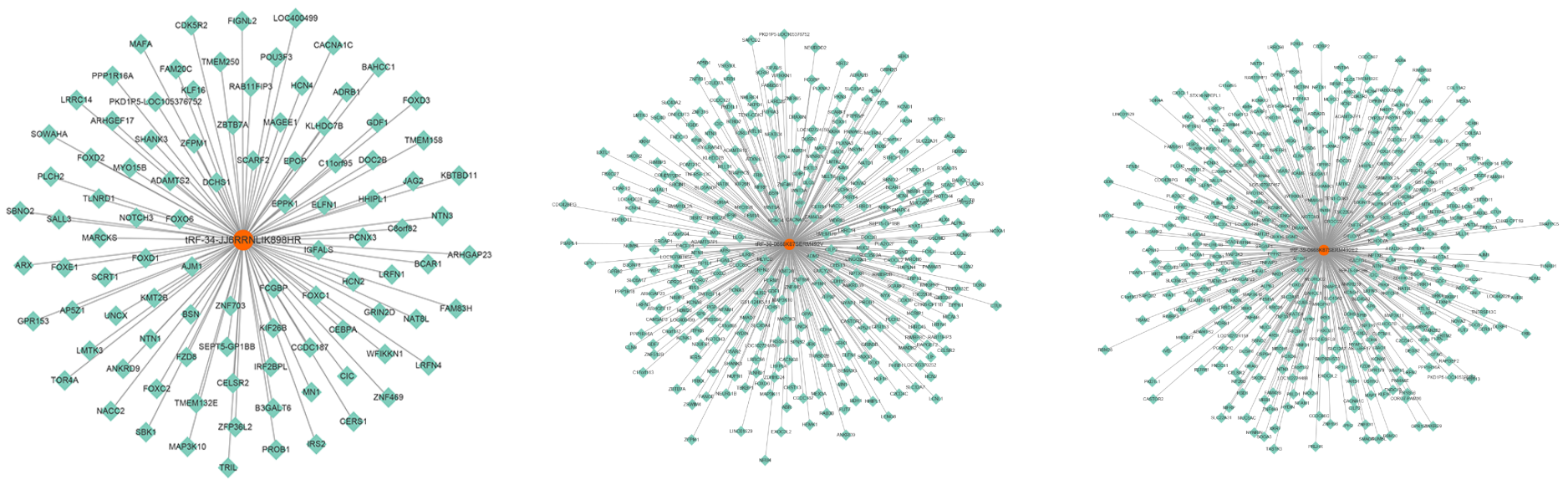

\section{Figure 4}

The target genes of differentially expressed tRFs. The orange color represents tRFs and green color represents target genes. A) tRF-34-JJ6RRNLIK898HR. B) tRF-38-0668K87SERM492V. C) tRF-39-0668K87SERM492E2. 

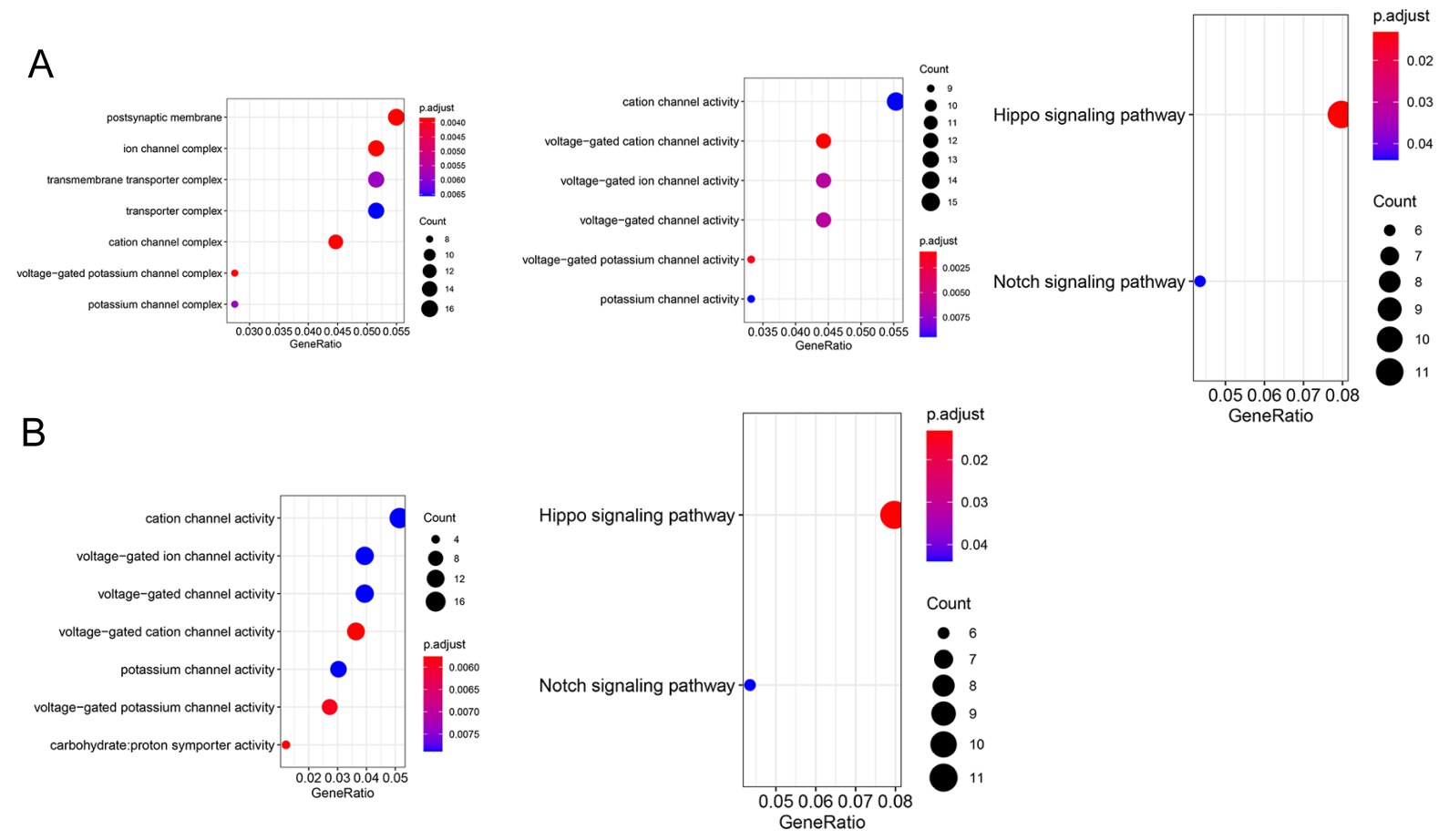

C
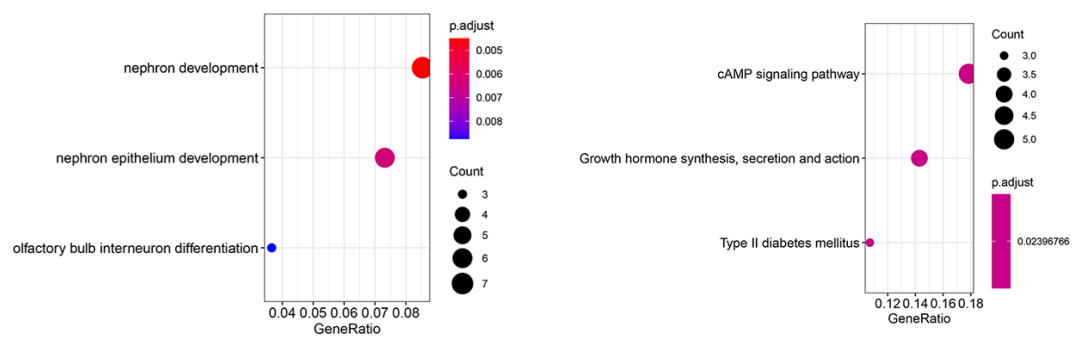

Figure 5

Bulb map of GO and KEGG analysis of dysregulated tRFs. Enrichment degree of target genes was showed by GeneRatio. The name of enrichment pathways was showed in $Y$ axis. The area of each node showed the number of the enriched target genes of differentially expressed tRFs (P<0.05). A) tRF-380668K87SERM492V. B) tRF-39-0668K87SERM492E2. C) tRF-34-JJ6RRNLIK898HR. GO, Gene Ontology; KEGG, Kyoto Encyclopedia of Genes and Genomes. 


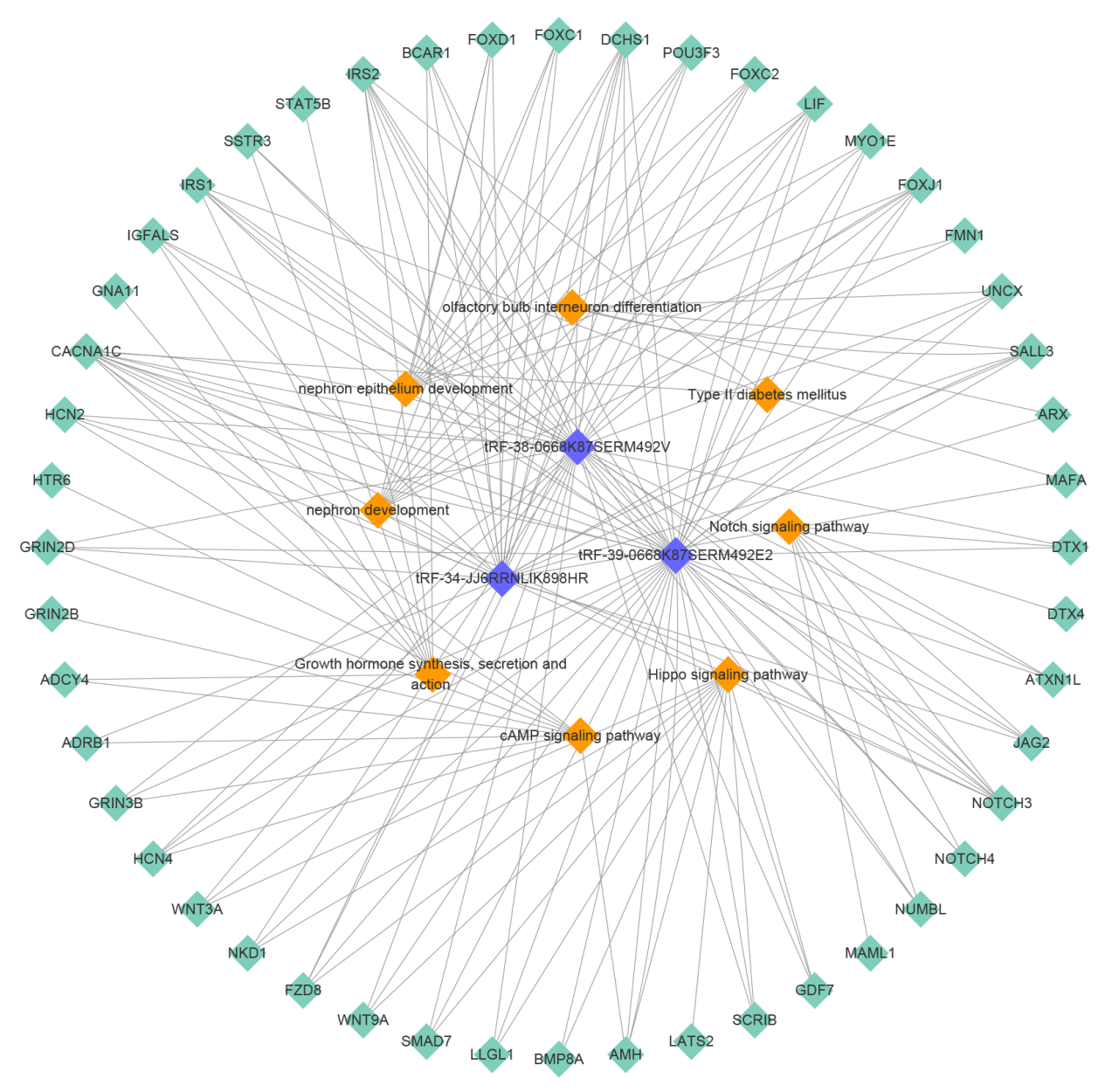

\section{Figure 6}

The relationship among differentially expressed tRFs, their target genes and related signaling pathways. Blue color represents differentially expressed tRFs, orange color represents signaling and green color represents target genes of tRFs. 

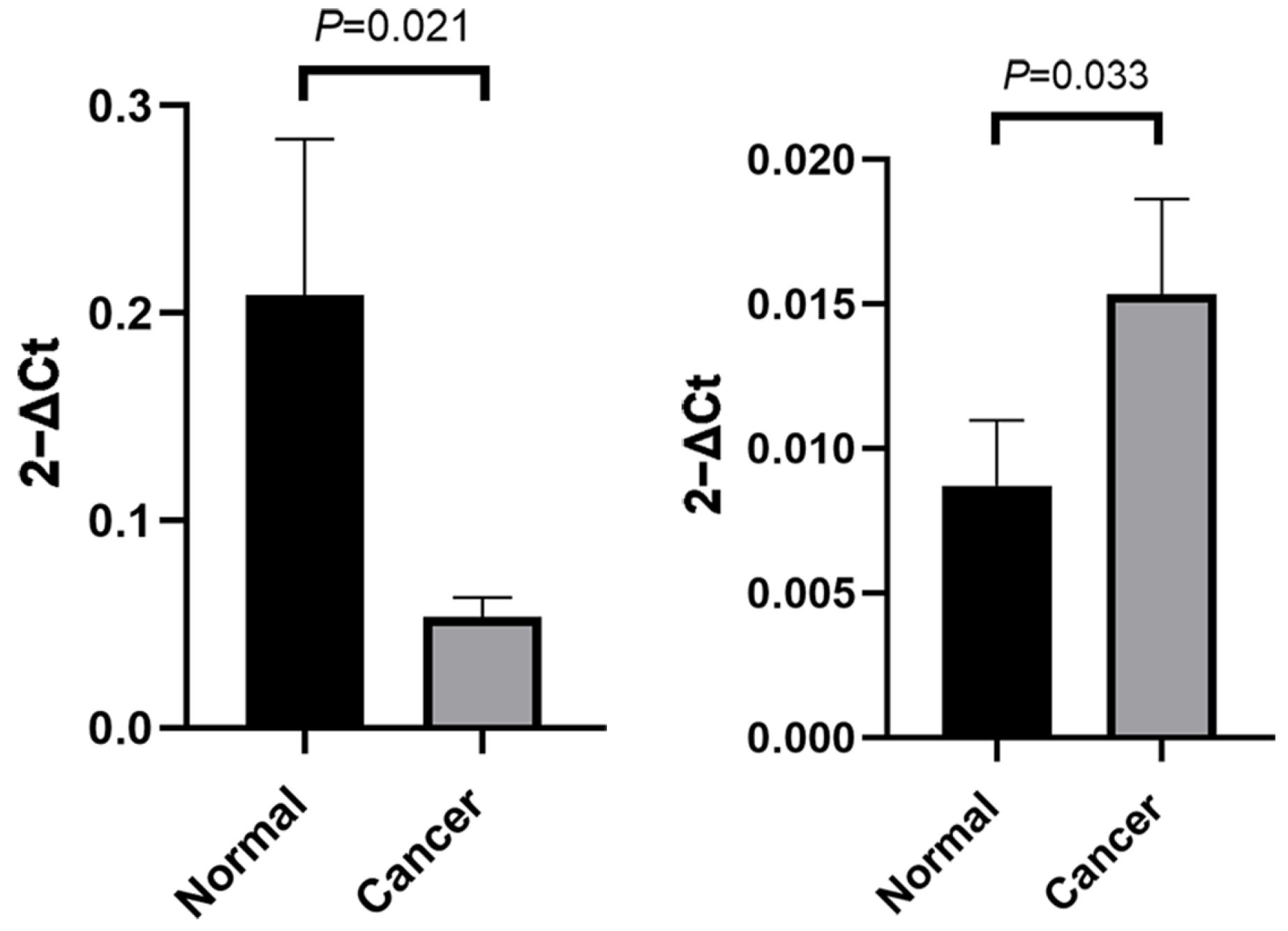

Figure 7

The relative expression of tRFs detected by qRT-PCR in CCA tissues $(P<0.05)$. Measurement data were expressed as mean \pm SEM. A) tRF-34JJ6RRNLIK898HR/tRF-38-0668K87SERM492V/tRF-39-0668K87SERM492E2. B) tRF-20-LE2WMK81. CCA, cholangiocarcinoma. qRT-PCR, quantitative realtime polymerase chain reaction. 
A

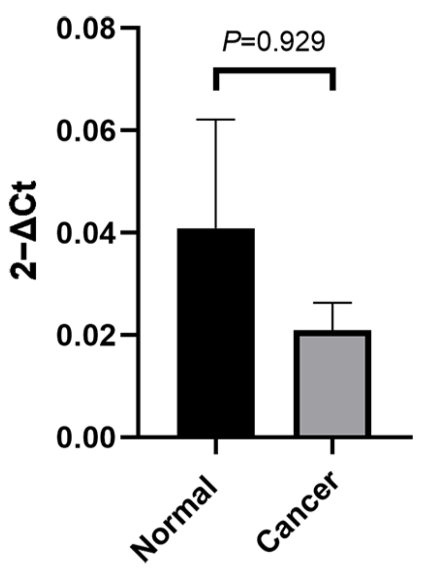

$\mathrm{E}$

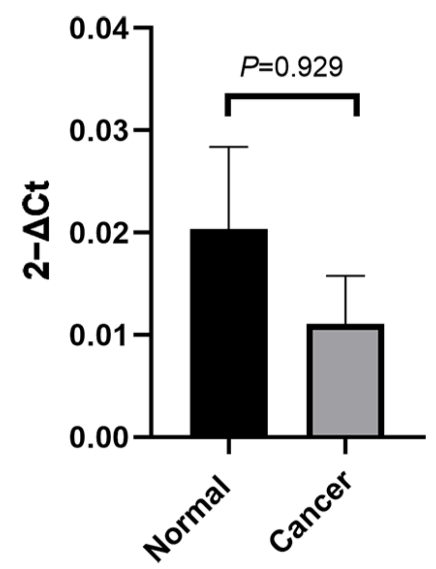

B

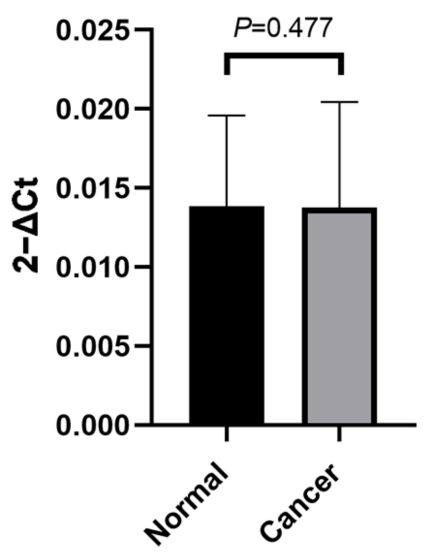

F

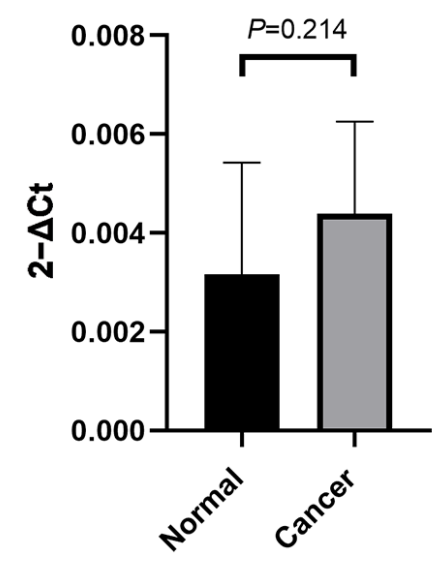

C
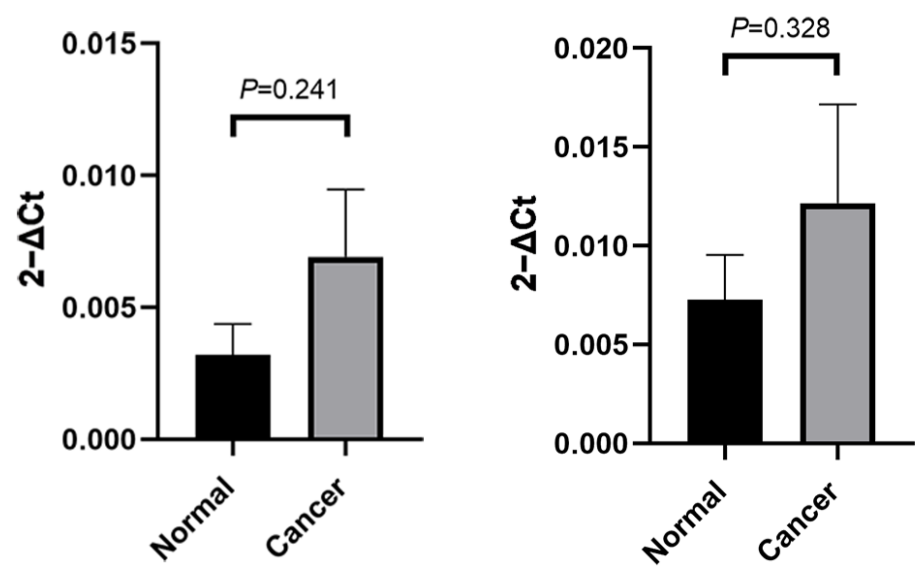

G

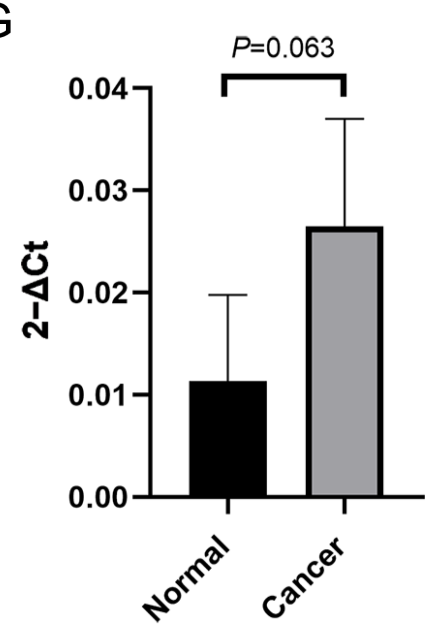

$\mathrm{H}$

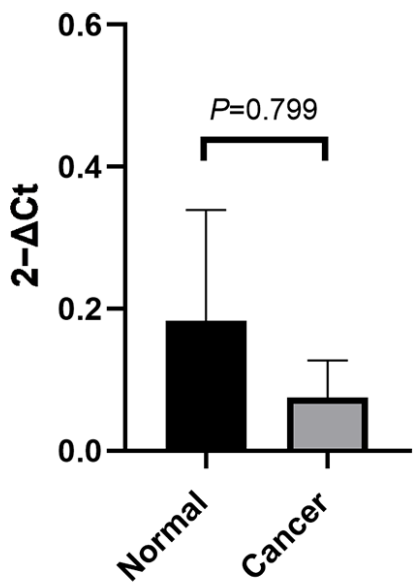

Figure 8

The relative expression of tRFs detected by qRT-PCR in CCA tissues( $P>0.05)$. Measurement data were expressed as mean \pm SEM. A) tRF-19-R118LOJX. B) tRF-19-BR29N3E2. C) tRF-21-RK9P4P9L0. D) tRF-16-9NF5W8B. E) tRF-16-KWEKK1B. F) tRF-17-K5KKOV2/tRF-18-P6KP6HD2. G) tRF-31-

6978WPRLXN4VE/tRF-22-8B8SOUPR2. H) tRF-18-1SS2P4X/tRF-17-YEKPRSP/tRF-17-ML5FX23/tRF-16-V0J809E CCA, cholangiocarcinoma. qRT-PCR, quantitative real-time polymerase chain reaction.

\section{Supplementary Files}

This is a list of supplementary files associated with this preprint. Click to download.

- supplementarymaterials.docx 